\title{
The potential and limitations of personalized medicine in the doctor-patient relationship
}

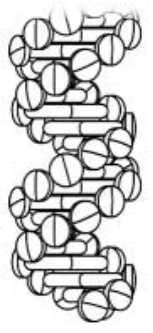

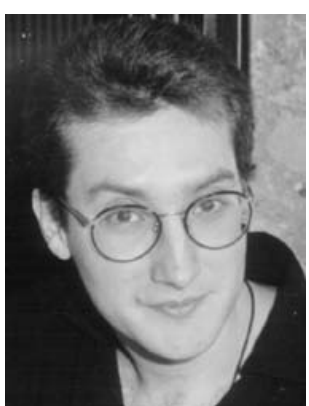

Rhydian H apgood, General Practitioner

Arnold $\mathrm{H}$ ealth $\mathrm{C}$ entre, Arnold, N ottingham, N G5 7BQ , UK

Tel: +44 (0)114 222 0776; Fax: +44 (0)114 222 0791; E-mail: r.hapgood@ sheffield.ac.uk
'The medic al world must be careful to avoid the genetic reductionist a pproach that is favored by the media where much is attributed to genetic variation.'

The UK G overnment's W hite Paper 'O ur Inheritance, Our Future' emphasizes, among other things, the potential benefits of pharmacogenetics to the doctor-patient relationship [101]. This commitment to a personalized medicine approach where genetics is used to tailor prescriptions to the individual is a very different model of care to the public health approach. Currently, treatment with most evidence for a given population is prescribed to 'try it and see'.

The possible benefits of prospective pharmacogenetic testing prior to drug prescription are almost unbelievable to the average general practitioner $(G P)$ and hold the potential to radically change the nature and content of a consultation. The prediction and avoidance of side effects may reduce the reluctance that many patients have towards taking medicines and may give doctors greater confidence in prescribing, knowing that they are less likely to cause harm. Equally, the faith that patients place in the prescription may be increased by the prediction of response to treatment. Individually tailored advice on lifestyle and disease prevention may make patients more likely to heed that advice and take healthy lifestyle choices. All these potential benefits may contribute to a more cost-effective health service.

Given these exciting benefits, it is significant that there is very little debate in the GP newspapers or academic press regarding pharmacogenetics. The major reason for this is that the technology promises much but appears to be delivering little. There is much published research on genetic microbiology, such as using genetics to monitor H IV resistance to antiretroviral medicines, and some research on genetic histology, particularly of cancers [1,2]. The best example of the latter is testing for HER2 status of a breast cancer in order to predict its responsiveness to transtuzumab chemotherapy. H owever, in a recent search of the literature, $48 \%$ of
M edline and EM BASE-cited entries for pharmacogenetics (defined as variation in drug response due to human genetics) were review articles, with only $11 \%$ of articles being original research examining the clinical validity of pharmacoge netic tests [3]. For a test to be useful clinically to a doctor and patient, the allele in question needs to have an association with change in a primary clinical end point of some kind, such as blood pressure. M oreover, that change needs to be of a magnitude that is not simply statistically significant but clinically significant, i.e., will change morbidity or mortality.

There are a small number of papers that demonstrate association between pharmacogenetics test results and primary clinical end points. Although this offers an enticing view of the future, the research fraternity needs to take a multidisciplinary view and be aware that it may bring new problems. For example, DNA is inherited, therefore, if an individual is unable to take a given drug, then it is likely that some family members will also be unable to do so. M oreover, the natural role of metabolic enzymes, the source of much pharmacogenetic variation, is to metabolize environmental toxins and carcinogens [4]. Therefore, it is possible that predictive disease associations with cancers may become apparent after a pharmacogenetics test has entered the marketplace. Given the M endelian nature of inheritance, then one individual having a pharmacogenetic test may unveil a cancer risk for his family members. This has clear clinical, ethical and health economic consequences.

'What is clear is that the costs and benefits of pharmacogenetic svary between c linic al situations.'

For some acute clinical situations, such as using codeine for pain relief or salbutamol for asthma, rather than having the economic expense of setting up a pharmacogenetic testing service it may be more cost-effective to simply try the drug and wait to see if it works [5-7]. For other situations, where drugs are used long-term and there is a high incidence of side effects, such 
as prescription of tricyclic antidepressants, it may be more prudent to start with a low dose of the drug and to titrate up slowly, rather than test for slow-metabolizer status [8]. W hat is clear is that the costs and benefits of pharmacogenetics vary between clinical situations and a good understanding of both clinical medicine and health economics are required in order to make the decision as to whether the pharmacogenetic approach is cost-effective for a given scenario.

Pharmacogenetic testing is likely to introduce new dimensions to the consultation and to make it longer, with more complex decisions. This will have economic and behavioral consequences. For example, the degree to which probabilities of side effects and efficacy are discussed will increase. Test results are probabilistic, not binary (i.e., there is a percentage chance of response/side effects). Informed choice regarding such treatment decisions will require the explanation of probabilistic test results to patients and decision rules probably based on health economics. Therefore, the information readily available to the doctor working at the coal face will need to be of a higher standard in order to ensure fully informed consent (the British $\mathrm{N}$ ational Formulary has no incidence or probabilities of this nature) [9]. Conversely, the medical world must be careful to avoid the genetic reductionist approach that is favored by the media where much is attributed to genetic variation. M ost problems dealt with by G Ps have several facets to them, including physical, psychological, social and spiritual. The holistic solution to a problem cannot be reduced to a single genetic test.

'Does hope have a measurable

utility to patients and, if so, how much is society willing to pay for it?'

An important point to consider is whether patients are likely to accept the label of nonresponder without trying a drug, particularly if this is a probabilistic not a binary label, and there may be no alternative treatment offered. Potential examples include transtuzumab for breast cancer and pravastatin for ischemic heart disease $[2,10]$. This raises the philosophical issue of the importance of the hope for a cure. Does hope have a measurable utility to patients and, if so, how much is society willing to pay for it?

Assessing the cost-effectiveness of a pharmacogenetic test requires consideration of the significant fixed costs of developing pharmacogenetics testing facilities and a skilled work force. In the UK this must be done by the $\mathrm{N}$ ational $\mathrm{H}$ ealth Service, while the $\mathrm{N}$ ational Institute for $\mathrm{Clinical}$ Excellence (NICE) will have to decide how to handle the uncertainty regarding the comparability of different genetic assays for the same genotype in economic models and by government licensing agencies. What D N A technology does offer NICE is a more personalized means of assessing the cost-benefit equation. Potentially this means that some individuals could get and some be refused the same treatment on explicitly defensible grounds rather than the current indefensible ones such as postcodes.

\section{'Pragmatic trials are needed in order to allow generalization of pharmacogenetic data to the real world of primary care.'}

Pharmacogenetics offers the pharmaceutical industry the chance to increase the probability of an ineffective drug getting a licence by defining a genetic subgroup in which it does work. H owever, it may be possible to increase the popularity of older drugs, such as tricyclics, if efficacious dose and adverse events were predictable [8]. This, together with fragmenting of the market place as populations are defined into genetic subgroups, may mean that pharmacogenetics reduces rather than increases profits for the industry. Genotyping prior to trials, to exclude individuals with a high risk of adverse events or to enrich trials with those who are more likely to respond, may reduce costs of drug development by allowing trial sizes to be smaller and more cost-effective [11]. However, such trials produce no evidence for efficacy in certain screened-out genotypes, and it is doubtful whether the results of such trials can be generalized to the population as a whole. M oreover, there is the thorny issue of polypharmacy and phenocopies (where a phenotype mimics a different genotype); for example, a genotypic fast metabolizer who requires a low dose of SSRI (selective serotonin reuptake inhibitor) for efficacy if given haloperidol (which blocks the enzyme concerned) becomes a phenotypic slow metabolizer prone to side effects. Clearly, pragmatic trials are needed in order to allow generalization of pharmacogenetic data to the real world of primary care. In order to determine the design of the trials, in particular the comparators and the variables to be measured, high quality health economic modeling is the essential precursor. 


\section{Bibliography}

Papers of special note have been highlighted as of interest $(\cdot)$ to readers.

1. Chaix C, Grenier-Sennelier C, Clevenbergh $\mathrm{P}$ : Economic evaluation of drug resistance genotyping for the adaptation of treatment in H IV-infected patients in the VIRAD APT study. J. Acquir. ImmuneD efic. Syndrome24, 227-231 (2000).

2. Vogel $C L$, Cobleigh M A, Tripathy $D$ et al.: Efficacy and safety of trastuzumab as a single agent in first-line treatment of HER2overexpressing metastatic breast cancer. J. Clin. Oncol. 20, 719-726 (2002).

3. H apgood R: A systematised search for evidence of 'real' pharmacogenetic tests. Society of Academic Primary C are Annual Scientific M eeting, M anchester (2003).

4. N ebert $D$, Ingelman-Sundberg M, D aly A: Genetic epidemiology of environmental toxicity and cancer susceptibility; human allelic polymorphisms in drug-metabolizing enzyme genes, their functional importance and nomenclature issues. D rug M etab. Rev. 31, 467-487 (1999).

5. Sindrup SH, Brosen K: The pharmacogenetics of codeine hypoal gesia. Pharmacogenetics 5, 335-46 (1995).

- $\quad$ Review with 91 refs.

6. D rysdale CM , M CG raw DW, Stack CB et al.: Complex promoter and coding region beta 2-adrenergic receptor haplotypes alter receptor expression and predict in vivo responsiveness. Proc. N atl. Acad. Sci. U SA 97,10483-10488 (2000).

7. Green SA, Rathz D A, Schuster AJ, Liggett SB: The lle164 beta(2)-adrenoceptor polymorphism alters salmeterol exosite binding and conventional agonist coupling to G(s). Eur. J. Pharmacol. 421, 141-147 (2001).

8. Kirchheiner J, Brosen $\mathrm{K}, \mathrm{D}$ ahl $\mathrm{ML}$ et al.: CYP2D 6 and CYP2C 19 genotype-based dose recommendations for antidepressants: a first step towards subpopulation-specific dosages. Acta Psychiatrica Scandinavica 104, 173-192 (2001).

9. BM A, RPSBG. British N ational Formulary. British M edical Association and the Royal Pharmaceutical Society of G reat Britain, London (2003).

10. Kuivenhoven JA, Jukema JW, Zwinderman $\mathrm{AH}$ et al.: The role of a common variant of the cholesteryl ester transfer protein gene in the progression of coronary atherosclerosis. The Regression Growth Evaluation Statin Study Group [see comments]. N. Engl. J. M ed. 338, 86-93 (1998).

11. Roses AD : Pharmacogenetics and the practice of medicine. $N$ ature $405,857-865$ (2000).

\section{- $\quad$ Review with 59 refs.}

\section{Website}

101. http://www.doh.gov.uk/genetics/ whitepaper.htm UK D epartment of $\mathrm{H}$ ealth G enetics W hite Paper. 